\title{
Atuação da Psicologia em Unidades de Terapia Intensiva
}

\section{Psychologist's Performance in Intensive Care Units}

\section{Mariane Silva Muniz ${ }^{\dagger}$ Bárbara Batista Silveira † $^{*}$}

Como citar esse artigo. Muniz, M.S,; Silveira, B.B. Atuação da Psicologia em Unidades de Terapia Intensiva. Revista Mosaico, v.11, n.2, p. 95 - 100, 2020.

Nota da Editora. Os artigos publicados na Revista Mosaico são de responsabilidade de seus autores. As informações neles contidas, bem como as opiniões emitidas, não representam pontos de vista da Universidade de Vassouras ou de suas Revistas.

\section{Resumo}

A psicologia hospitalar é um campo de atuação da psicologia da saúde que busca tratar os aspectos psicológicos em torno do adoecimento. Este artigo de revisão da literatura especializada tem como objetivo analisar a atenção psicológica prestada em UTI aos pacientes, familiares e a equipe multidisciplinar. Para tanto, foi realizada uma pesquisa, através da busca ativa de informações em livros, revistas e artigos acadêmicos, especificamente nas bases Scielo, Medline, Lilacs, PePSIC, PsycINFO voltados ao tema em questão. A partir do levantamento da literatura revisada, destacamos que o papel do psicólogo(a) no contexto da UTI é ser um agente de mudanças, um especialista em relações, aquele que reúne conhecimentos e técnicas para uma atuação voltada para a atividade curativa e preventiva, tendo a função de restabelecer o estado de saúde do doente, de seus familiares e de auxiliar a equipe de saúde.

Palavras-chave: Psicologia Hospitalar. Psicólogo. UTI.

\section{Introdução}

A psicologia hospitalar é um campo de atuação da psicologia da saúde que busca tratar os aspectos psicológicos em torno do adoecimento. A Unidade de Terapia Intensiva (UTI) é caracterizada como um dos cenários mais agressivos e traumáticos do hospital, principalmente por se apresentar como um espaço de confronto entre a vida e a morte. A internação em UTI causa um impacto enorme ao paciente, seus familiares e à equipe de saúde. (ALMEIDA; MALAGRIS, 2011)

A internação em uma UTI exige atenção especial por se tratar de um ambiente que oferece cuidado a pacientes em condições críticas. A inserção do(a) profissional de psicologia nesse contexto é fundamental para esses indivíduos, pois tem o objetivo de propiciar uma melhor compreensão da situação e dos sentimentos por ele desencadeados. Além de atuar com o paciente e seus familiares, o(a) psicólogo(a) também é de grande valia para toda a equipe multidisciplinar de saúde que atua neste cenário, pois se trata de um ambiente com uma alta carga de estresse e a atuação do psicólogo(a) pode contribuir para uma melhora do relacionamento entre a equipe, bem como da equipe com os pacientes/ familiares. (SILVA, 2010)

Este trabalho torna-se relevante por mostrar que a internação na UTI pode trazer consequências emocionais para aqueles que fazem parte desse cenário: pacientes, familiares e profissionais e, diante disso, insere-se a importância das intervenções do(a) Psicólogo(a) com os referidos grupos. Desta forma, responderemos ao seguinte questionamento: como o(a) profissional da Psicologia atua na Unidade de Terapia Intensiva?

\footnotetext{
Afiliação dos autores:

†Psicóloga, Vassouras, RJ, Brasil

\$ Professora na Universidade de Vassouras, Vassouras, RJ, Brasil
}

* Email de correspondência: barbie.silveira@hotmail.com 
A fim de responder tal questionamento, este artigo de revisão da literatura especializada teve como objetivo analisar a atenção psicológica prestada em UTI aos pacientes, familiares e a equipe multidisciplinar, conceituando Psicologia da Saúde e Psicologia Hospitalar, identificando como funciona uma Unidade de Terapia Intensiva e analisando a importância do Psicólogo(a) neste contexto.

Para tanto, foi realizada uma pesquisa, através da busca ativa de informações em livros, revistas e artigos acadêmicos, especificamente nas bases Scielo, Medline, Lilacs, PePSIC, PsycINFO voltados ao tema em questão.

\section{A Psicologia da Saúde}

Historicamente, a origem da Psicologia da Saúde ocorreu no ano de 1970 através da American Psychological Association (APA) com um grupo de psicólogos(as) interessado no trabalho do âmbito da saúde, para buscar compreender o processo saúdedoença. (CASTRO; BORNHOLD, 2004)

Segundo Martins (2001), a Psicologia da Saúde surge então da necessidade de promover e de pensar o processo saúde/doença como um fenômeno social. Ainda, de acordo com o autor, o interesse dos(as) psicólogos(as) no contexto da saúde, se deu pela saída desses profissionais das clínicas privadas, contribuindo assim, na inserção desses profissionais na área pública.

Complementando, Castro e Bornholdt (2004) inferem que a Psicologia da Saúde em seu principal objetivo, vai além da compreensão de como os fatores biológicos e comportamentais influenciam no processo saúde-doença, considerando também os fatores sociais.

No Brasil, a Psicologia tem sido inserida nas políticas de saúde a partir do processo de implantação do Sistema Único de Saúde (SUS). A ciência psicológica integra-se ao campo da saúde no momento em que seu campo de compreensão, transposto das ciências físico-naturais, em que as leis da natureza explicavam o fenômeno humano, encontra-se com as relações que os humanos travam consigo mesmos e com os outros, escapando às leis da natureza. (FOUCAULT, 2002)

Corrobora Foucault (2002) que o crescimento da psicologia da saúde pode ser atribuído a três fatores:

a) à evidência do aumento de doenças e da mortalidade, causadas pelo estilo de vida;

b) ao fortalecimento da filosofia dos países industrializados de que os indivíduos são responsáveis por sua própria saúde; e

c) ao aumento da discordância com o modelo médico e sua dominância na atenção à saúde.

Neste contexto, podemos inferir que a Psicologia da Saúde pode ser vista como um campo da Psicologia que nasce para dar soluções aos problemas envolvidos no processo saúde-doença. Sebastiani (2003) destaca algumas tendências para essa área, tais como: integração de modelos teóricos, modificação de crenças a atitudes com relação às enfermidades, a participação individual e comunitária nas questões de saúde.

Desta forma, é necessário enfatizar que a Psicologia da Saúde ressalta as intervenções no âmbito social e inclui aspectos que vão além do trabalho no hospital. Neste estudo, também abordaremos a Psicologia Hospitalar, visto que o foco do trabalho é esmiuçar a atuação do(a) psicólogo(a) nas Unidades de Terapia Intensiva, um complexo hospitalar. Porém, vale destacar que Castro e Bornholdt (2004) afirmam que a Psicologia Hospitalar é apenas uma estratégia de atuação em Psicologia da Saúde, embora no Brasil, infelizmente, a identidade do(a) psicólogo(a) esteja vinculada ao lugar em que atua, entende-se que o marco conceitual da Psicologia da Saúde é a que deve servir de base para a Psicologia Hospitalar.

\section{A Psicologia Hospitalar}

Os hospitais podem serdefinidos como instituições complexas dentro do campo da saúde, que fazem uso de novas e aprimoradas tecnologias, com o objetivo de responder às transformações vivenciadas nesse campo. É um dos serviços designados à produção de ações de saúde para atender às necessidades dos pacientes e seus familiares. Para que as atividades sejam realizadas nesse contexto, há extensa divisão de trabalho entre os profissionais e um sistema de coordenação de tarefas e funções. (CRP, 2016)

Segundo Pimentel et al. (2009) os primeiros registros de inserção do(a) psicólogo(a) no âmbito hospitalar foi no ano de 1818 quando, no Hospital McLean, em Massachussets, formou-se a primeira equipe multiprofissional que incluía o(a) psicólogo(a). E em 1904 foi fundado, neste mesmo hospital, um laboratório de Psicologia, onde foram desenvolvidas as primeiras pesquisas sobre Psicologia Hospitalar.

A Psicologia Hospitalar é o campo de entendimento e tratamento dos aspectos psicológicos em torno do adoecimento, aquele que se dá quando o sujeito humano, carregado de subjetividade, esbarra em um real, de natureza patológica, denominado doença. Foi reconhecida como especialidade em $2001 \mathrm{e}$, atualmente, regulamentada pela Resolução do Conselho Federal de Psicologia no 13/2007. Esta Resolução institui normas e procedimentos para a atuação dos(as) Psicólogos(as) nos hospitais e defender as peculiaridades da atuação nesse ambiente. (SIMONETTI, 2004)

Segundo a Resolução CFP n ${ }^{\circ}$ 013/2007, a Psicologia Hospitalar, tem a função de desenvolver atividades em diferentesníveis de tratamento, tendo como sua principal tarefa a avaliação e o acompanhamento 
de intercorrências psíquicas dos pacientes que estão ou serão submetidos a procedimentos médicos, visando basicamente à promoção e/ou a recuperação da saúde física e mental.

A Psicologia Hospitalar objetiva dar voz à subjetividade aproximando-se do paciente em sofrimento, favorecendo a elaboração simbólica do adoecimento, a travessia do tratamento necessário e trabalhando no sentido de validar sentimentos presentes entrando em contato com as dificuldades do momento que pode parecer insuportável e infinito. (CFP, 2019)

A Psicologia Hospitalar é uma área do conhecimento que visa fornecer suporte ao sujeito em adoecimento, com o intuito de que este possa atravessar essa fase com maior resiliência. É um campo de entendimento e tratamento dos aspectos biológicos e psicológicos em torno do adoecimento e não somente doenças psicossomáticas. Desta forma, podemos inferir que a Psicologia Hospitalar é uma área inovadora, que pode atuar em diversos setores, sendo que os casos mais comuns de inserção do(a) psicólogo(a) na equipe multidisciplinar são: maternidade, emergência, centro cirúrgico, pronto socorro, UTI e CTI. (CHIATTONE, 2011).

\section{A Unidade de Terapia Intensiva}

A Unidade de Terapia Intensiva (UTI) é um ambiente onde são prestados cuidados intensivos a pacientes em estado grave ou que necessitam de um cuidado mais rigoroso por parte dos profissionais da saúde. (MACHADO, 2004)

A Criação da UTI aconteceu em meados do século XX, nos Estados Unidos, e veio se evidenciando como unidade indispensável aos doentes graves. No Brasil, a UTI também surgiu no século XX, mais precisamente na década de 1970, no auge do milagre econômico que, no contexto, privilegiava um modelo econômico concentrador de renda e uma política voltada à modernização e ao desenvolvimento, o que repercutiu no setor da saúde em que a expansão se deu às custas da ação do Estado. (GUSMÃO, 2012)

O Ministério da Saúde define a UTI como sendo um local onde existe um conjunto de elementos funcionalmente agrupados, estando destinados ao atendimento de pacientes graves ou de risco que exijam assistência médica e de enfermagem ininterruptas, além de equipamentos e recursos humanos especializados e consiste na combinação de cuidado intensivo de enfermagem com constante atenção médica na assistência prestada ao paciente crítico, reduzindo a morbidade e a mortalidade sempre que possível. (BRASIL, 1998)

Em seus estudos, Gusmão (2012) relata que, segundo a Associação de Medicina Intensiva Brasileira (AMIB), os médicos e a equipe de enfermagem compõem o quadro principal de profissionais da unidade; porém, outras áreas de saúde foram inseridas como importantes auxiliares do tratamento do paciente crítico, incluindose a Psicologia (GUSMÃO, 2012).

Complementando, Gusmão (2012) explicita que a UTI é formada por uma equipe multidisciplinar composta por diversos profissionais da saúde, os quais devem oferecer uma assistência 24 horas por dia aos pacientes. Os profissionais que trabalham nessa unidade são: médicos, enfermeiros, farmacêuticos, terapeutas ocupacionais, fisioterapeutas, nutricionistas, psicólogos(as) e assistentes sociais, que podem ter sua esfera psicológica impactada pela morte podendo encará-la como fracasso pessoal.

Pregnolatto e Agostinho (2010) inferem que a UTI é um setor diferenciado de outras unidades de um hospital, pois oferece tratamento específico e intensivo para o paciente em estado crítico. Ainda segundo os autores, a UTI é um ambiente hostil, invasivo e impacta negativamente a saúde mental dos sujeitos que são submetidos à internação, bem como de familiares e da equipe de saúde.

Oliveira (2002) infere que a UTI, em sua generalidade, é um local onde se internam pacientes graves que ainda têm um prognóstico favorável para viver. Ainda, segundo o autor, nesse local são atendidos casos de pessoas que se encontram em uma situação limite (entre a vida e a morte) e necessitam de recursos técnicos e humanos especializados para sua recuperação.

A UTI é caracterizada por dispor de uma aparelhagem de cuidado altamente complexa e os profissionais que nela trabalham são técnicos especializados em cuidados específicos. Assim, é um espaço onde os pacientes críticos estão internados, gerando uma certa angústia e preocupação aos familiares, amigos e até para os profissionais da saúde que atuam neste espaço, pois a doença afeta biopsicossocialmente o sujeito que está ali internado (MACHADO, 2004).

De acordo com Machado (2004) a partir do aumento das tecnologias em exames, máquinas e evolução no conhecimento das medicações, a UTI tem cada vez mais condições de cuidado para o paciente em estado grave, pós-cirúrgico. Durante muitos anos, o acesso à UTI para famílias e amigos era restrito, até mesmo para os profissionais da saúde; poucos circulavam dentro desse espaço, em função do cuidado com a contaminação e infecções.

Segundo Costa, Figueiredo e Schaurich (2009), geralmente, em uma UTI a tecnologia tende a se sobrepor aos fatores ligados ao cuidado, pois a equipe de saúde que atua neste ambiente fica envolvida com as máquinas e os monitores e tende a se esquecer que, atrás da doença, existe um paciente e sua família. Desse contexto, nasce a necessidade da inserção da Psicologia na UTI, visando refletir sobre o atendimento psicológico 
ao paciente crítico, com o objetivo de reconhecer os meios e o processo de um tratamento invasivo em que o paciente se submete para garantir a sua vida.

\section{Atuação do(a) Psicólogo(a) na UTI}

No hospital, a Psicologia tem como principal função o acolhimento e o trabalho com pacientes de todas as faixas etárias, bem como seus familiares, em sofrimento psíquico decorrente de suas patologias, internações e tratamentos. Nesse processo, é fundamental reconhecer que o(a) Psicólogo(a) encontra-se em uma realidade muito diferente da vivenciada no consultório, pois no hospital a atuação desse profissional é pautada pela instituição, na medida em que está exposta a um conjunto de regras, valores, rotinas, dinâmica acelerada e repleta de acontecimentos inesperados. (LAZARETTI et al., 2007).

Segundo a Resolução CFP no 013/2007 o(a) psicólogo(a) atua em instituições de saúde na prestação de serviços nos pontos secundário ou terciário da atenção à saúde. A definição da especialidade prevê que o(a) psicólogo(a) hospitalar:

[...] Atende a pacientes, familiares e/ou responsáveis pelo paciente; membros da comunidade dentro de sua área de atuação; membros da equipe multiprofissional e eventualmente administrativa, visando o bem-estar físico e subjetivo do paciente; e, alunos e pesquisadores, quando estes estejam atuando em pesquisa e assistência. Oferece e desenvolve atividades em diferentes níveis de tratamento, tendo como sua principal tarefa a avaliação e acompanhamento de intercorrências psíquicas dos pacientes que estão ou serão submetidos a procedimentos médicos, visando basicamente a promoção e/ou a recuperação da saúde física e mental. Promove intervenções direcionadas à relação médico/paciente, paciente/família, e paciente/paciente e do paciente em relação ao processo do adoecer, hospitalização e repercussões emocionais que emergem neste processo (CFP, 2007, p. 21).

Nesta perspectiva, o(a) psicólogo(a) busca intervir como mediador psicológico, buscando compreensão na relação entre equipe/paciente e equipe/família. A equipe e a família exercem papéis importantes durante o processo de relação do paciente com a sua doença, sendo que o paciente irá compartilhar suas limitações, dificuldades, medos, dependências, impotências, sentimento de culpa, negação da realidade e dificuldades em adaptar-se à rotina hospitalar (PIMENTEL; LIMA; FONSECA, 2009).

De acordo com Gusmão (2012) a inserção da Psicologia nas UTI tem o intuito de oferecer suporte ao paciente crítico e seus familiares e apoio à equipe interdisciplinar, no sentido de proporcionar a todos uma percepção das dimensões biopsicossociais da saúde, do adoecer e da morte humanizados.
$\mathrm{Na}$ UTI, onde o risco de vida e a possibilidade da morte estão presentes, o psicólogo pode facilitar e/ ou favorecer o curso da vida; a isto se pode denominar promoção de saúde e de qualidade de vida. Neste sentido, a Psicologia Hospitalar situa-se além do trabalho de humanização da instituição, oferecendo tratamento específico para as questões do ser humano no decorrer da sua história de vida (LAZARETTI et al., 2007, p. 10).

Desta forma, o(a) psicólogo(a) na UTI estará implicado não só com o sofrimento, mas com a evolução do quadro clínico até a fase final, com o luto e o óbito. Deste modo, é importante saber: quem é a pessoa assistida? Qual é a patologia? Aguda ou crônica? Com sequelas provisórias ou definitivas? Está devidamente informado sobre seu tratamento? E a família? Participativa? Fragilizada? Quais as repercussões psicossociais na vida da pessoa assistida e da sua família? A doença já evoluiu para a fase terminal? Pode-se fazer paliação? (CFP, 2019)

Segundo CRP (2016), no que refere-se à organização do trabalho na UTI, os atendimentos são realizados a partir de solicitação de consulta ou busca ativa, de maneira que o(a) Psicólogo(a) precisa se deslocar até o leito do paciente e, nesse, estabelecer o setting terapêutico. Além disso, quando é solicitada intervenção psicológica a um paciente, há que se considerar que ela poderá ser breve, por vezes única, pois dependerá do tempo de internamento de cada paciente.

$\mathrm{O}$ (a) Psicólogo(a) também deve estar advertido(a) com relação à multiplicidade de solicitações, não apenas pela rotatividade de pacientes que pode ser muito alta, mas principalmente por estar em jogo a angústia de toda uma equipe. Desta forma, destaca o CRP (2016) que o(a) psicólogo(a) deve atuar junto aos agentes envolvidos no processo de hospitalização na UTI com os seguintes objetivos:

- Orientar e informar rotinas da UTI e horário de visita;

- Informar ao paciente acerca dos acontecimentos que ocorrem fora da UTI, mesmo o paciente estando em coma (inconsciente);

- Estimular o contato do paciente com a família e equipe de saúde, objetivando a facilitação da comunicação;

- Avaliar a compreensão do quadro clínico e prognóstico por familiares e paciente.

Corrobora Lazaretti et al. (2007), que o(a) Psicólogo(a) na UTI atua como um facilitador da comunicação e da expressão humana através da linguagem, visando a representação e a elaboração das vivências em internamentos ou adoecimentos.

Em seus estudos Romaro (2008) explicita que o trabalho do(a) Psicólogo(a) precisa ter como ponto de partida o fato de que, embora o paciente esteja passando por um momento crítico de adoecimento e internação, 
ele continua tendo uma identidade e precisa ser parte ativa em todo o seu tratamento. Desta forma, toda intervenção psicológica com os pacientes visa elaborar as possíveis consequências do impacto relacionado ao adoecer, à internação e ao tratamento, bem como aos possíveis agravamentos decorrentes, trabalhando o resgate da esperança e da luta pela vida. Ainda, segundo Romaro (2008) todas essas ações capacitarão o paciente a lidar com as situações difíceis referentes ao seu quadro clínico, além das possibilidades e limites do tratamento.

Sendo assim, a atuação do(a) psicólogo com paciente internado na UTI tem como principal objetivo identificar características de funcionamento psíquico normal ou patológico nas condições de uma patologia e quais as intervenções pertinentes a qualquer uma destas condições. A condição crítica do paciente exige que o(a) psicólogo(a) leve em consideração os aspectos clínicos de cada caso de forma bastante criteriosa, pois só assim será possível separar quadros psíquicos de quadro orgânicos e definir condutas. Para atuar é necessário conhecer bem os fundamentos da Psicologia do Desenvolvimento, do processo psíquico envolvido no adoecer, da psicopatologia, do processo de morte e das intervenções (CFP, 2019).

Junto à equipe multiprofissional, o(a) Psicólogo(a) tem o papel de colaborar para o aprimoramento da comunicação, auxiliar a tomada de decisões e encaminhamentos frente às dificuldades ou queixas apresentadas pelos pacientes e/ou familiares, podendo o(a) Psicólogo(a) mediar a relação entre equipe/paciente e equipe/equipe, a fim de que o ambiente hospitalar seja menos estressor para os profissionais e usuários. (CRP, 2016)

Complementando, Lazaretti et al. (2007) enfatiza que outra função do(a) Psicólogo(a) junto à equipe, é realizar um trabalho de escuta com os profissionais de saúde, intervindo nos conflitos e sofrimentos. Nesta perspectiva, segundo estes autores, é necessário zelar pela posição de integrante da equipe, o que exige muitas vezes, que essa escuta seja realizada por um profissional externo à equipe.

No âmbito familiar, o(a) Psicólogo(a) pode oferecer suporte psicológico, já que frente ao adoecimento e hospitalização de um integrante desse grupo, a família pode manifestar as mais variadas reações, desde se opor ao tratamento como até ser uma importante rede de apoio e auxiliar nas estratégias de enfrentamento desenvolvidas pelo paciente. $\mathrm{O}(\mathrm{A})$ Psicólogo(a) também pode mediar a relação família/ paciente e/ou família/equipe, a fim de que o ambiente hospitalar se torne menos hostil para a família (CRP, 2016).

Nesta perspectiva, vale ressaltar que, no contexto da UTI, tanto os familiares quanto a equipe de saúde poderão ser assistidas pela Psicologia, devido às dificuldades no processo de reabilitação ou na iminência da perda, pois o adoecer provoca, na maioria das vezes, inúmeras alterações psicológicas. Desta forma, segundo Moreira, Martins e Castro (2012) cabe ao psicólogo(a) escutar e observar todos os aspectos ligados ao adoecer, respeitando os temores, as crenças e as fragilidades do paciente, de seus familiares e até mesmo da equipe de saúde.

A prática clínica psicológica na UTI acontece dirigida ao ser que adoece frente às diversas patologias e os diferentes espaços de atendimento. Onde houver sofrimento desadaptações, otrabalho do(a)psicólogo(a) incide nos aspectos subjetivos e emocionais do adoecimento, assim como nos possíveis desdobramentos relacionados ao tratamento, recuperação, sequelas, cuidados paliativos e óbito. (CFP, 2019)

Concluindo, o(a) psicólogo(a) na UTI deve ter atenção a todas essas questões citadas anteriormente. Sua função junto ao paciente e seus familiares é diagnosticar seu estado emocional, estimular o vínculo do paciente com o tratamento, auxiliar no enfrentamento da dor, do sofrimento, da angústia e da morte, direcionar sobre questões práticas e minimizar os agentes estressores e negativos que retardam a reabilitação do paciente. Junto à equipe de saúde, o(a) psicólogo(a) tem o objetivo de assegurar-lhes informações sobre a família e o doente que possam vir a interferir no tratamento. Outro ponto importante é a promoção do fortalecimento do vínculo paciente-família-médico, sendo um intermediário nas comunicações que possam estar dificultadas. Cabe também ao(a) psicólogo(a) identificar as demandas emocionais da equipe de saúde visando o acolhimento destas. (CFP, 2019)

\section{Considerações Finais}

Neste trabalho, foi possível perceber que a Unidade de Tratamento Intensivo (UTI) é um ambiente de cuidados da saúde, com potencial de gerar estados emocionais que podem interferir na evolução do paciente, na vida de seus familiares e nas equipes de trabalho, já que a morte é vista como fracasso e tabu, mesmo pelos profissionais de saúde.

A partir do levantamento da literatura revisada, destacamos a importância da atuação do(a) Psicólogo(a) na UTI, para os pacientes, familiares e equipe de saúde multiprofissional. Desta forma, a atuação do(a) Psicólogo(a) na UTI abrange a promoção do enfrentamento da doença por todos esses personagens.

Com relação aos objetivos desta revisão, foi possível identificar que a Psicologia Hospitalar pode ser entendida como um conjunto de contribuições profissionais que as diferentes disciplinas psicológicas priorizam para dar assistência integral aos pacientes hospitalizados. O papel do(a) psicólogo(a) no contexto 
da UTI é ser um agente de mudanças, um especialista em relações, aquele que reúne conhecimentos e técnicas com a atuação voltada para a atividade curativa e preventiva, tendo a função de restabelecer o estado de saúde do doente, de seus familiares e de auxiliar a equipe de saúde.

Desta forma, podemos inferir que o trabalho do(a) psicólogo(a) na UTI é fazer com que o paciente tenha um cuidado integral, com suas subjetividades e particularidades respeitadas dentro de suas demandas e possibilidades, além de apoiar e informar a família dos processos que o indivíduo enfermo está passando, ou seja passar a informação de uma maneira menos técnica, buscando entender o sofrimento e a doença, orientando como será o tratamento e evolução do caso, auxiliando os colegas profissionais da saúde como fazer este cuidado de forma interdisciplinar, onde todos os envolvidos neste processo buscam a melhor qualidade de vida ou de morte deste paciente, e assim realizar os encaminhamentos para as redes de cuidado conforme a necessidade do paciente e da família.

Neste contexto, o(a) psicólogo(a), quando atua em um espaço como a UTI, fica diante da concretude da experiência vivida e participa dos fatos que se transformarão em acontecimentos na vida do paciente e de seus familiares, assim como da construção dos elos da cadeia de signos da história pessoal de cada um.

Por fim, espera-se que este trabalho desperte interesse e incentive a realização de novos estudos nesta área. Portanto, relata-se a necessidade de futuras pesquisas que abordem em específico a atuação do(a) Psicólogo(a) na UTI.

\section{Referências}

ALMEIDA, R. A.; MALAGRIS, L. E. N. A Prática da Psicologia da Saúde. Revista da SBPH, 14(2), 183-202, 2011

BRASIL. Ministério da Saúde. Secretaria de Vigilância Sanitária. Portaria n ${ }^{\circ}$ 466, de 04 de junho de 1998. Regulamento Técnico para o funcionamento dos serviços de tratamento intensivo. Diário Oficial da União. Brasília. 1998.

CASTRO, E. K.; BORNHOLDT, E. Psicologia da Saúde x Psicologia Hospitalar: Definições e possibilidades de inserção profissional. Revista Psicologia ciência e profissão, Brasília, v. 24, n. 3, set. 2004.

COSTA, S. C., FIGUEIREDO, M. R., SCHAURICH, D. Humanização em Unidade de Terapia Intensiva Adulto (UTI): compreensões da equipe de enfermagem. Interface, 13(suppl.1), 571-580, 2009.

CFP. Conselho Federal de Psicologia (Brasil). Resolução N. ${ }^{\circ}$ 013/2007. Institui a Consolidação das Resoluções relativas ao Título Profissional de Especialista em Psicologia e dispõe sobre normas e procedimentos para seu registro. Brasil. 2007.

CFP. Conselho Federal de Psicologia (Brasil). Referências técnicas para atuação de psicólogos(as) nos serviços hospitalares do SUS. Conselho Federal de Psicologia, Conselhos Regionais de Psicologia e Centro de Referência Técnica em Psicologia e Políticas Públicas. Brasília: CFP, 2019.

CHIATTONE, H. B. C. A Significação da Psicologia no Contexto Hospitalar. Psicologia da Saúde - um novo significado para a prática clínica. 2. ed. rev. amp. São Paulo: Cengage Learning, 2011. p. 145-233.

CRP. Caderno de Psicologia Hospitalar: Considerações sobre Assistência,
Ensino, Pesquisa e Gestão. Bruno Jardini Mäder (org.). Curitiba : CRP-PR, 2016.

FOUCAULT, M. A Psicologia de 1850 a 1950. In: FOUCAULT, M. A. (org.). Ditos e Escritos I (pp. 133-151). Rio de Janeiro: Forense Universitária. 2002.

GUSMÃO, L. M. Psicologia Intensiva: nova especialidade. 2012. Disponível em: http://www.redepsi.com.br/2012/05/08/psicologia-intensiva-novaespecialidade/. Acesso em: 15 Fev. 2020

LAZARETTI, C. et al. Manual de Psicologia Hospitalar. CRP-PR. Curitiba: Unificado, 2007. Coletânea Conexão Psi.

MACHADO, E. G. de A. Enfermagem em Unidade de Terapia Intensiva Goiânia: Cultura e Qualidade, 2004.

MARTINS, D. G. Psicologia da saúde e o novo paradigma: Novo paradigma? Revista Psicologia Teoria e Prática, São Paulo, v. 3, n. 1, maio 2001. Disponível em: http://editorarevistas.mackenzie.br/index.php/ptp/article/ view/1098. Acesso em: 10 Set. 2019

MOREIRA, E. K. C. B., MARTINS, T. M., CASTRO, M. M. Representação Social da Psicologia Hospitalar para Familiares de Pacientes Hospitalizados em Unidade de Terapia Intensiva. Revista SBPH, 15(1), 134-167, 2012.

OLIVEIRA, E. C. N. O psicólogo na UTI: reflexões sobre a saúde, vida e morte nossa de cada dia. Psicologia: Ciência e Profissão, Brasília, v. 22, n. 02, p.102-119, 2002.

PIMENTEL, D. M.; LIMA, D. T.; FONSECA, R. M. AAtuação do Psicólogo Hospitalar no Atendimento aos Portadores de Câncer de Próstata e de Mama. Trabalho de conclusão de curso. Faculdade de Ciências Humanas e Sociais da Universidade do Vale do Rio Doce. Curso de Graduação em Psicologia. Governador Valadares, jun. 2009.

PREGNOLATTO, A. P. F.; AGOSTINHO, V. B. M. O psicólogo na unidade de terapia intensiva - adulto. In: BAPTISTA, M. N. Psicologia Hospitalar: Teoria, Aplicações e Casos Clínicos. 2. ed. Rio de Janeiro: Guanabara Koogan, 2010.

ROMARO, R. A. Intervenções e psicoterapia breve no contexto hospitalar In: LANGE, E. S. N. (org.) Contribuições à psicologia hospitalar: desafios e paradigmas. São Paulo: Vetor, 2008 p. 75-91.

SEBASTIANI, R. W. Psicologia da Saúde no Brasil: 50 Anos de História. 2003.

SILVA, A. J. S. et al. Assistência de Enfermagem na UTI: Uma Abordagem Holística. Revista Eletrônica de Enfermagem do Centro de Estudo de Enfermagem e Nutrição, v. 1, n. 1, p. 1-16, 2010. Disponível: https:// studylibpt.com/doc/4806163/assist $\%$ C3\%AAncia-de-enfermagem-na-uti-uma-abordagem-hol\%C3\%ADstica. Acesso: Acesso em: 10 Set. 2019.

SIMONETTI, A. Manual de Psicologia Hospitalar. São Paulo: Casa do Psicólogo, 2004. 